\title{
Preliminary genome-wide association mapping of rice bacterial leaf blight resistance loci using major Korean races of Xoo (Xanthomonas oryzae)
}

\author{
Asjad Ali ${ }^{1}$, Do Yoon-Hyun ${ }^{1}$, Tae-Hwan Noh ${ }^{2}$, Yu-Mi Choi ${ }^{1}$, Sukyeung Lee ${ }^{1}$, Sejong Oh$^{1}$, Myung-Chul \\ Lee $^{1^{*}}$
}

${ }^{1}$ National Agrobiodiversity Center, National Institute of Agricultural Sciences, RDA, Jeonju 54874, Republic of Korea

${ }^{2}$ National Institute of Crop Science (NICS), RDA, 181, Iseo-myeon, Wanju, Jeollabuk-do, 55365, Korea

"Corresponding author: mcleekor@korea.kr

\begin{abstract}
Bacterial leaf blight (BLB), caused by X. oryzae pv. oryzae (Xoo), is one of the most destructive diseases of rice due to its high epidemic potential. Understanding BLB resistance at a genetic level is important to further improve the rice breeding that provides one of the best approaches to control BLB disease. In the present investigation, a collection of 96 accessions was used in the genomewide association study (GWAS) for BLB resistance loci against four Korean races of Xoo that were represented by the prevailing BLB isolates under Xoo differential system. The results of the bioassay using a selected set of 96 accessions showed that a large number of accessions $(93.75 \%)$ were resistant to K1 race, while the least number of accessions (34.37\%) resisted K3a race. For races $\mathrm{K} 2$ and $\mathrm{K} 3$, the resistant germplasm proportion remained between 66.67 to $70.83 \%$. The genotypic data produced SNP matrix for a total of 293,379 SNPs. After imputation the missing data was removed, which exhibited 34,724 SNPs for association analysis. GWAS results showed strong signals of association at a threshold of $[-\log 10(P$-value) $]$ more than 5 (K1 and K2) and more than 4 (K3 and K3a) for nine of the 39 SNPs, which are plausible candidate loci of resistance genes. These SNP loci were positioned on rice chromosome 2, 9, and 11 for $\mathrm{K} 1$ and $\mathrm{K} 2$ races, whereas on chromosome 4, 6, 11, and 12 for K3 and K3a races. The significant loci detected have also been illustrated, NBS-LRR type disease resistance protein, SNARE domain containing protein, Histone deacetylase 19, NADP-dependent oxidoreductase, and other expressed and unknown proteins. Our results provide a better understanding of the distribution of genetic variation of BLB resistance to Korean pathogen races and breeding of resistant rice cultivars.
\end{abstract}

Keywords: Bacterial leaf blight, rice, GWAS, SNP, X. oryzae.

Abbreviations: BLB_bacterial leaf blight; CDS_coding sequence; DAI_days after inoculation; GWAS_genome-wide association study; LRR_leucine rich repeat; PSA_peptone sucrose agar; QTL_quantitative trait loci; SNP_single nucleotide polymorphism; UTR_untranslated region.

\section{Introduction}

The productivity of rice is limited by pathogens such as Xanthomonas oryzae pv. Oryzae (Xoo), which is a causal agent of bacterial leaf blight (BLB) of rice. BLB is one of the disastrous diseases that lead to crop failure in tropical and temperate rice growing regions of the world (Mew, 1987; Khan et al., 2014). In Korea, BLB appeared as an emerging disease in past years, affecting yield and grain quality of rice (Noh et al., 2007). Various studies have been carried out related to disease management and control. However, enhancing genetic resistance has proven to be the most effective method of controlling BLB disease. A total of 38 BLB resistance genes ( $R$ genes), designated as $\mathrm{Xa1}$ to $\mathrm{Xa38}$, have been identified in rice (Khan et al., 2014; Kim et al., 2016). These R genes evoke a strong, normally race-specific, resistance that results in very short lesions and reduction of susceptibility. However, the pathogen populations rapidly evolve as indicated by the emergence of various pathotypes and races to overcome the resistance. Finding new sources of durable resistance is a continuing challenge for effective control of bacterial leaf blight. The dominant $\mathrm{R}$ genes include Xa1, Xa2, Xa3/Xa26, Xa4, Xa6, Xa7, Xa10, Xa11, Xa12, Xa14, Хa16, Xa17, Xa18,
$X a 21, X a 22(t), X a 23, X a 25, X a 27, X a 29(t), X a 30(t), X a 32(t)$, $X a 35(t)$, and $X a 36(t)$ and the recessive $\mathrm{R}$ genes include $x a 5$, $x a 8, x a 9, x a 13, x a 15, x a 19, x a 20, x a 24, x a 25 / X a 25(t), x a 26(t)$, $x a 28(t), x a 31(t), x a 33(t)$, and $x a 34(t)$ (Chen et al., 2011; Khan et al., 2014). Several BLB genes have been mapped on chromosome 4 (Xa1, Xa2, Xa12, Xa14, and Xa25), chromosome 5 (xa5), chromosome 6 (Xa7), chromosome 8 (xa13), and chromosome 11 (Xa3, Xa4, Xa10, Xa21, Xa22, and Xa23) (Chen et al., 2002; Das et al., 2014; Khan et al., 2014). The location of the remaining genes is not clear at the moment. Seven recessive genes $(x a 5, x a 8, x a 13, x a 24, x a 26, x a 28$, and xa32) occur naturally and confer race-specific resistance, whereas three recessive genes, including $x a 15, x a 19, x a 20$ are the product of mutagenesis and confer broad spectrum of resistance to Xoo races (Lee et al., 2003; Ogawa, 1996). Several BLB resistance genes have been physically mapped and characterized for their spectrum of resistance. The gene Xal was identified by Sakaguchi (1967) that confers specific resistance to race 1 strain of Xoo in Japan. It encodes a nucleotide binding LRR protein (Yoshimura et al., 1998). Xa3 gene has been mapped to the long arm of chromosome 11 and 
tightly linked to Xa4 gene (Yoshimura et al., 1992). It has been reported that plants carrying $\mathrm{Xa} 3$ gene showed resistance to nine Xoo races in Philippines (Zhang et al., 1998). Similarly, $\mathrm{Xa2l}$ was reported as a highly effective gene against South and Southeast Asian races of Xoo (Khush et al., 1990). Xa26 is also a dominant gene that codes for a LRR receptor kinase protein. It has been mapped on the long arm of chromosome 11 and found in cultivar Mingui 63 that was resistant to many strains of Xoo during seedling and adult stages (Chen et al., 2002; Taura et al., 1992). Similarly, Xa27 gene conferred resistance against a wide range of Xoo strains.

The distinctness of Xoo populations from different Asian countries has been reported on the basis of molecular genotyping studies covering diversity, distribution of pathogens and relationship between phylogeny and virulence (Nelson et al., 1994; Adhikari et al., 1995). Based on the population structure, Korean Xoo races are disparate from the Philippines and Japanese that might be due to continuous genetic variations in isolates and their unique hierarchical evolutionary pathways for pathogenicity (Jeung et al., 2006). In Korea, these isolates have been grouped into five races (K1 to K5). However, the main focus has been concentrated on the $\mathrm{K} 1, \mathrm{~K} 2, \mathrm{~K} 3$, and $\mathrm{K} 3 \mathrm{a}$ races (Noh et al., 2003; Jeung et al., 2006). Among these races, $\mathrm{K} 1$ effect is declining due to rice cultivars bearing $\mathrm{XaI}$ and $\mathrm{Xa3}$ gens, whereas $\mathrm{K} 2$ and $\mathrm{K} 3$ races have increased their pathogenicity in Korea. In particular, recently evolved race K3a caused a severe damage in the southwestern areas of Korea (Noh et al., 2003).

Traditional gene mapping methods have been used to identify and localize target genes in many crops. Though these mapping methods using $\mathrm{F}_{2}$ populations and recombinant inbred lines are useful in targeting genes, but these are time consuming and provide low mapping resolution. Genome-wide association (GWA) mapping is a technique that links the specific phenotype to sequence variation present in the individual's genome at various loci (Nordborg and Weigel, 2008). In comparison to traditional methods, GWA uses natural populations to rapidly map the target genes in large and diverse genotypes with much higher resolution. Recently, GWAS has been reported for the analysis of complex traits in foxtail millet, rice, sorghum, and maize (Huang et al., 2010; Kump et al., 2011; Jia et al., 2013). GWAS was also used for the identification of genes linked to complex traits such as leaf size, flowering time and disease resistance (Buckler et al., 2009; Poland et al., 2011).

Single nucleotide polymorphism (SNP) chips have been used in GWAS to identify genes and QTL linked traits in rice such as abiotic stress, grain quality, and agronomic performance. In our research, we examined BLB resistance in GWAS based on genotyping SNPs variants across diverse accessions of rice. The goal of this study was, using GWAS, to identify a considerable number of loci related to BLB resistance that might be important for rice improvement.

\section{Results}

\section{Resistance reaction to Xoo races}

Ninety-six accessions collected from 11 different countries were tested for their resistance to four Korean races of Xoo. The resistance patterns for the four isolates have been shown on the representative leaves of rice accessions in Fig. 1. The highest resistance in terms of number of accessions was observed against K1 race (93.75\%) followed by K3 (70.83\%), and K2 (66.67\%) races. The most prevailing threat to rice in Korea, K3a race showed a more devastating effect on germplasm with high susceptibility (65.62\%) (Fig. 2, Table 1).
In total, 22 accessions expressed resistance to all four races of Xoo. The germplasm from the Philippines was more resistant with 11 accessions, followed by China and Korea with 4 and 3 accessions, respectively. However, single accession from each of Japanese (IT123177) and American (IT226) germplasm showed resistance against four races. All the germplasm accessions held resistance genes for at least one of the pathogen races. Furthermore, sixteen accessions were observed with susceptibility to three races with more shares from Japan (13 accessions). Among the Korean germplasm, all accessions showed resistance against $\mathrm{K} 1$ and $\mathrm{K} 3$ races, whereas nine out of thirteen accessions were resistant to K2 race. However, only three accessions (IT260672, IT219216, and IT219282) were resistant to $\mathrm{K} 3 \mathrm{a}$ race and interestingly, these accessions also showed resistance to other three races (K1, K2, and K3) of the pathogen (Table 1).

\section{GWAS for resistance to BLB strains}

By employing the data set of 34,724 high quality SNPs, the GWAS revealed 8, 17, 4 and 10 SNPs associated with BLB against pathogen at a threshold of $[-\log 10(P$-value $)]$ more than 5 for $\mathrm{K} 1$ and $\mathrm{K} 2$, and more than 4 for $\mathrm{K} 3$ and $\mathrm{K} 3 \mathrm{a}$, respectively. Among them, the strongest trait associated SNPs (or linear peak SNPs) for each race were selected and considered as the putative loci for BLB resistance in rice. A total of nine putatively BLB linked SNP loci were identified on different chromosomes. Four loci for K1 and K2 races were declared to have a highly significant association with BLB resistance. These associated loci were located on three chromosomes of rice holding their positions on chromosome 9 and 11 for K1 race (Fig. 3a) and 2 and 11 for K2 race (Fig. 3b). Similarly, 5 loci for $\mathrm{K} 3$ and $\mathrm{K} 3 \mathrm{a}$ races were detected in connection with BLB resistance. The linked loci were positioned on chromosome 6 and 11 for K3 race (Fig. 3c) and chromosome 4, 11 and 12 for K3a race (Fig. 3d). Manhattan plots of four races showed different patterns of SNPs distribution from each other except chromosome 11, which holds the significant SNPs for $\mathrm{K} 1, \mathrm{~K} 2, \mathrm{~K} 3$ and $\mathrm{K} 3 \mathrm{a}$ races but at variable peaks. Three peaks with $-\log 10(P$-value) values larger than 7 (Fig. 3a, 3b) and two peaks with $-\log 10(P$-value) values larger than 5 (Fig. 3c, 3d) in Manhattan plots indicated very strong signals of association between the trait and chromosomal regions. In particular, five regions on chromosome 9 (Fig. 3a), 2 (Fig. 3b), 6 (Fig. 3c), 4 and 12 (Fig. 3d) host sharp -log10( $P$-value) peaks.

A set of 39 SNPs was detected from the whole dataset with variable $-\log 10(P$-value $)$ values on different chromosomal locations for all four races of the pathogen. Among these BLB associated (putative) SNPs, 16 were located in CDS region, 14 in intergenic, and 7 in the intron region of the annotated genes, whereas two SNPs were present in the UTR region of LOC_Os11g38480 and LOC_Os02g57520 (Table 2). MLM analysis detected the highest number of SNPs for race K2 followed by $\mathrm{K} 3 \mathrm{a}, \mathrm{K} 1$ and $\mathrm{K} 3$ races with seventeen, ten, eight, and four SNPs, respectively. In approximately $1.70 \mathrm{Mb}$ interval (27090877 - 28791142) on rice chromosome 11, seven BLB associated (putative) SNPs were located in the CDS, intron and intergenic regions of seven putative genes. Among these genes, the CDS region genes at LOC_Os11g46250 have been annotated as expressed proteins while the function of other four putative BLB linked intergenic regions is unknown. Similarly, two SNPs were located in approximately $0.1 \mathrm{Mb}$ interval (22801669 - 22908256) on chromosome 11 in UTR and CDS regions at LOC_Os11g38480 and LOC_Os11g38630, which have been annotated as NBS-LRR type disease resistance and expressed proteins, respectively (Table 2 ).

The sequences of the newly identified loci were blasted using 
Table 1. Details of 96 accessions of rice used in this study and their response to four Korean races of Xoo.

\begin{tabular}{|c|c|c|c|c|c|c|c|c|c|c|c|c|c|c|c|c|c|c|c|c|}
\hline \multirow{2}{*}{ No. } & \multirow{2}{*}{$\begin{array}{l}\text { Accession } \\
\text { number }\end{array}$} & \multirow{2}{*}{$\begin{array}{l}\text { Country } \\
\text { of origin }\end{array}$} & \multicolumn{4}{|c|}{ Resistance/susceptibility $^{\mathrm{a}}$} & \multirow{2}{*}{ No. } & \multirow{2}{*}{$\begin{array}{l}\text { Accession } \\
\text { number }\end{array}$} & \multirow{2}{*}{$\begin{array}{l}\text { Country of } \\
\text { origin }\end{array}$} & \multicolumn{4}{|c|}{ Resistance/susceptibility } & \multirow{2}{*}{ No. } & \multirow{2}{*}{$\begin{array}{l}\text { Accession } \\
\text { number }\end{array}$} & \multirow{2}{*}{$\begin{array}{l}\text { Country of } \\
\text { origin }\end{array}$} & \multicolumn{4}{|c|}{ Resistance/susceptibility } \\
\hline & & & K1 & K2 & K3 & K3a & & & & K1 & $\mathrm{K} 2$ & K3 & $\mathrm{K} 3 \mathrm{a}$ & & & & K1 & $\mathrm{K} 2$ & K3 & $\mathrm{K} 3 \mathrm{a}$ \\
\hline 1 & IT66 & Japan & $\mathrm{R}$ & $\mathrm{S}$ & $S$ & $\mathrm{~S}$ & 33 & K177617 & Japan & $\mathrm{R}$ & $\mathrm{R}$ & $\mathrm{R}$ & $\mathrm{S}$ & 65 & IT102195 & Philippines & $\mathrm{R}$ & $\mathrm{R}$ & $\mathrm{R}$ & $\mathrm{R}$ \\
\hline 2 & IT1387 & Japan & $\mathrm{S}$ & $\mathrm{R}$ & S & S & 34 & IT3242 & Japan & $\mathrm{R}$ & $\mathrm{R}$ & $\mathrm{S}$ & S & 66 & IT217976 & Philippines & $\mathrm{R}$ & $\mathrm{R}$ & $\mathrm{R}$ & $\mathrm{R}$ \\
\hline 3 & IT1624 & Japan & $\mathrm{R}$ & $\mathrm{S}$ & $\mathrm{R}$ & S & 35 & IT1141 & Japan & S & $\mathrm{s}$ & $\mathrm{R}$ & $\mathrm{S}$ & 67 & IT219962 & Philippines & $\mathrm{R}$ & $\mathrm{R}$ & $\mathrm{R}$ & $\mathrm{R}$ \\
\hline 4 & IT3192 & Japan & $\mathrm{R}$ & $\mathrm{S}$ & S & S & 36 & IT219963 & China & $\mathrm{R}$ & $\mathrm{R}$ & $\mathrm{R}$ & $\mathrm{R}$ & 68 & IT248324 & Philippines & $\mathrm{R}$ & $\mathrm{R}$ & $\mathrm{R}$ & $\mathrm{R}$ \\
\hline 5 & IT3290 & Japan & $\mathrm{R}$ & $\mathrm{R}$ & $\mathrm{R}$ & S & 37 & K037785 & China & $\mathrm{R}$ & $\mathrm{R}$ & $\mathrm{S}$ & $\mathrm{S}$ & 69 & IT122849 & Philippines & $\mathrm{R}$ & $\mathrm{R}$ & $\mathrm{R}$ & $\mathrm{R}$ \\
\hline 6 & IT3307 & Japan & $\mathrm{R}$ & $\mathrm{S}$ & $\mathrm{R}$ & S & 38 & IT260503 & China & $\mathrm{R}$ & $\mathrm{R}$ & $\mathrm{R}$ & S & 70 & IT284191 & Philippines & $\mathrm{R}$ & $\mathrm{R}$ & $\mathrm{S}$ & $\mathrm{R}$ \\
\hline 7 & IT3752 & Japan & $\mathrm{R}$ & $\mathrm{S}$ & $\mathrm{R}$ & $\mathrm{R}$ & 39 & IT291365 & China & $\mathrm{R}$ & $\mathrm{R}$ & $\mathrm{R}$ & $\mathrm{S}$ & 71 & IT284194 & Philippines & $\mathrm{R}$ & $\mathrm{R}$ & $\mathrm{R}$ & $\mathrm{S}$ \\
\hline 8 & IT5496 & Japan & $\mathrm{R}$ & $\mathrm{S}$ & $\mathrm{S}$ & S & 40 & IT223671 & China & $\mathrm{R}$ & $\mathrm{R}$ & $\mathrm{R}$ & $\mathrm{R}$ & 72 & IT284237 & Philippines & $\mathrm{R}$ & $\mathrm{R}$ & $\mathrm{R}$ & $\mathrm{R}$ \\
\hline 9 & IT5868 & Japan & $\mathrm{S}$ & $\mathrm{S}$ & $\mathrm{R}$ & $\mathrm{R}$ & 41 & IT223672 & China & $\mathrm{R}$ & $\mathrm{R}$ & $\mathrm{R}$ & $\mathrm{R}$ & 73 & IT259443 & USA & $\mathrm{R}$ & $\mathrm{R}$ & $\mathrm{R}$ & $\mathrm{S}$ \\
\hline 10 & IT6326 & Japan & $\mathrm{R}$ & $\mathrm{S}$ & $\mathrm{R}$ & $\mathrm{R}$ & 42 & IT223796 & China & $\mathrm{R}$ & $\mathrm{R}$ & $\mathrm{R}$ & $\mathrm{R}$ & 74 & IT226 & USA & $\mathrm{R}$ & $\mathrm{R}$ & $\mathrm{R}$ & $\mathrm{R}$ \\
\hline 11 & IT6614 & Japan & $\mathrm{R}$ & $\mathrm{S}$ & $\mathrm{R}$ & $\mathrm{R}$ & 43 & IT266274 & Korea & $\mathrm{R}$ & $\mathrm{S}$ & $\mathrm{R}$ & S & 75 & IT219234 & India & $\mathrm{R}$ & $\mathrm{R}$ & $\mathrm{R}$ & $\mathrm{S}$ \\
\hline 12 & IT6628 & Japan & $\mathrm{S}$ & $\mathrm{R}$ & $\mathrm{R}$ & S & 44 & K115659 & Korea & $\mathrm{R}$ & $\mathrm{R}$ & $\mathrm{R}$ & $\mathrm{S}$ & 76 & K177612 & India & $\mathrm{R}$ & $\mathrm{R}$ & $\mathrm{R}$ & $\mathrm{S}$ \\
\hline 13 & IT6668 & Japan & $\mathrm{R}$ & $\mathrm{S}$ & $\mathrm{R}$ & $\mathrm{R}$ & 45 & IT260672 & Korea & $\mathrm{R}$ & $\mathrm{R}$ & $\mathrm{R}$ & $\mathrm{R}$ & 77 & IT3457 & Puerto Rico & $\mathrm{R}$ & $\mathrm{S}$ & $\mathrm{S}$ & $\mathrm{S}$ \\
\hline 14 & IT7413 & Japan & $\mathrm{S}$ & $\mathrm{S}$ & $\mathrm{R}$ & S & 46 & IT191961 & Korea & $\mathrm{R}$ & $\mathrm{s}$ & $\mathrm{R}$ & $\mathrm{S}$ & 78 & K128330 & Indonesia & $\mathrm{R}$ & $\mathrm{R}$ & $\mathrm{R}$ & $\mathrm{S}$ \\
\hline 15 & IT7664 & Japan & $\mathrm{S}$ & $\mathrm{R}$ & $\mathrm{S}$ & S & 47 & IT212543 & Korea & $\mathrm{R}$ & $\mathrm{R}$ & $\mathrm{R}$ & $\mathrm{S}$ & 79 & IT268022 & Colombia & $\mathrm{R}$ & $\mathrm{R}$ & $\mathrm{R}$ & $\mathrm{S}$ \\
\hline 16 & IT123190 & Japan & $\mathrm{R}$ & $\mathrm{S}$ & $\mathrm{R}$ & S & 48 & IT219225 & Korea & $\mathrm{R}$ & $\mathrm{S}$ & $\mathrm{R}$ & $\mathrm{S}$ & 80 & IT265529 & Myanmar & $\mathrm{R}$ & $\mathrm{R}$ & $\mathrm{R}$ & $\mathrm{S}$ \\
\hline 17 & IT211133 & Japan & $\mathrm{R}$ & $\mathrm{R}$ & $S$ & S & 49 & IT266613 & Korea & $\mathrm{R}$ & $\mathrm{R}$ & $\mathrm{R}$ & $\mathrm{S}$ & 81 & IT3976 & Bolivia & $\mathrm{R}$ & $\mathrm{S}$ & $\mathrm{R}$ & $\mathrm{S}$ \\
\hline 18 & IT214732 & Japan & $\mathrm{R}$ & $\mathrm{R}$ & $\mathrm{R}$ & S & 50 & IT192004 & Korea & $\mathrm{R}$ & $\mathrm{R}$ & $\mathrm{R}$ & S & 82 & IT265585 & Unknown & $\mathrm{R}$ & $\mathrm{S}$ & $\mathrm{S}$ & $S$ \\
\hline 19 & IT247895 & Japan & $\mathrm{R}$ & $\mathrm{R}$ & S & S & 51 & K115114 & Korea & $\mathrm{R}$ & $\mathrm{R}$ & $\mathrm{R}$ & S & 83 & K125790 & Unknown & $\mathrm{R}$ & $\mathrm{R}$ & $\mathrm{R}$ & $S$ \\
\hline 20 & IT8869 & Japan & $\mathrm{R}$ & $\mathrm{S}$ & $\mathrm{R}$ & S & 52 & K115412 & Korea & $\mathrm{R}$ & $\mathrm{s}$ & $\mathrm{R}$ & $\mathrm{S}$ & 84 & IT7576 & Unknown & $\mathrm{R}$ & $\mathrm{S}$ & $\mathrm{S}$ & $\mathrm{S}$ \\
\hline 21 & IT264259 & Japan & $\mathrm{R}$ & $\mathrm{S}$ & $S$ & S & 53 & IT219216 & Korea & $\mathrm{R}$ & $\mathrm{R}$ & $\mathrm{R}$ & $\mathrm{R}$ & 85 & K175522 & Unknown & $\mathrm{R}$ & $\mathrm{R}$ & $\mathrm{R}$ & $\mathrm{S}$ \\
\hline 22 & IT123177 & Japan & $\mathrm{R}$ & $\mathrm{R}$ & $\mathrm{R}$ & $\mathrm{R}$ & 54 & IT219282 & Korea & $\mathrm{R}$ & $\mathrm{R}$ & $\mathrm{R}$ & $\mathrm{R}$ & 86 & IT149923 & Unknown & $\mathrm{R}$ & $\mathrm{R}$ & $\mathrm{S}$ & $\mathrm{S}$ \\
\hline 23 & IT204 & Japan & $\mathrm{R}$ & $\mathrm{S}$ & $\mathrm{R}$ & $\mathrm{R}$ & 55 & IT251353 & Korea & $\mathrm{R}$ & $\mathrm{R}$ & $\mathrm{R}$ & $\mathrm{S}$ & 87 & K168592 & Unknown & $\mathrm{R}$ & $\mathrm{R}$ & $\mathrm{S}$ & $\mathrm{S}$ \\
\hline 24 & IT2570 & Japan & $\mathrm{R}$ & $\mathrm{S}$ & $\mathrm{R}$ & $\mathrm{R}$ & 56 & K115177 & Philippines & $\mathrm{R}$ & $\mathrm{R}$ & $\mathrm{R}$ & $\mathrm{S}$ & 88 & 18154 & Unknown & $\mathrm{R}$ & $\mathrm{R}$ & $\mathrm{R}$ & $S$ \\
\hline 25 & IT100829 & Japan & $\mathrm{R}$ & $\mathrm{S}$ & $\mathrm{R}$ & $\mathrm{R}$ & 57 & IT9771 & Philippines & $\mathrm{R}$ & $\mathrm{R}$ & $\mathrm{S}$ & S & 89 & K034669 & Unknown & $\mathrm{R}$ & $\mathrm{R}$ & $\mathrm{R}$ & $\mathrm{R}$ \\
\hline 26 & IT10063 & Japan & $\mathrm{R}$ & $\mathrm{S}$ & S & S & 58 & IT9820 & Philippines & $\mathrm{R}$ & $\mathrm{R}$ & $\mathrm{R}$ & $\mathrm{R}$ & 90 & K034671 & Unknown & $\mathrm{R}$ & $\mathrm{R}$ & $\mathrm{R}$ & S \\
\hline 27 & IT10236 & Japan & $\mathrm{R}$ & $\mathrm{S}$ & S & S & 59 & IT268017 & Philippines & $\mathrm{R}$ & $\mathrm{R}$ & $\mathrm{R}$ & $\mathrm{R}$ & 91 & K041462 & Unknown & $\mathrm{R}$ & $\mathrm{R}$ & $\mathrm{R}$ & $\mathrm{R}$ \\
\hline 28 & IT 10078 & Japan & $\mathrm{R}$ & $\mathrm{S}$ & $S$ & S & 60 & IT260462 & Philippines & $\mathrm{R}$ & $\mathrm{R}$ & $\mathrm{R}$ & $\mathrm{S}$ & 92 & IT9417 & Unknown & $\mathrm{R}$ & $\mathrm{R}$ & $\mathrm{s}$ & $\mathrm{R}$ \\
\hline 29 & IT 10287 & Japan & $\mathrm{R}$ & $\mathrm{S}$ & S & S & 61 & IT265428 & Philippines & $\mathrm{R}$ & $\mathrm{R}$ & $\mathrm{R}$ & $\mathrm{R}$ & 93 & IT9465 & Unknown & $\mathrm{R}$ & $\mathrm{R}$ & $\mathrm{S}$ & $\mathrm{R}$ \\
\hline 30 & IT 10486 & Japan & $\mathrm{R}$ & $\mathrm{S}$ & S & S & 62 & IT265436 & Philippines & $\mathrm{R}$ & $\mathrm{R}$ & $\mathrm{R}$ & $\mathrm{S}$ & 94 & IT9533 & Unknown & $\mathrm{R}$ & $\mathrm{R}$ & $\mathrm{S}$ & $\mathrm{S}$ \\
\hline 31 & IT7987 & Japan & $\mathrm{R}$ & $\mathrm{S}$ & S & S & 63 & IT101863 & Philippines & $\mathrm{R}$ & $\mathrm{R}$ & $\mathrm{R}$ & $\mathrm{R}$ & 95 & IT219259 & Unknown & $\mathrm{R}$ & $\mathrm{R}$ & $\mathrm{S}$ & $S$ \\
\hline 32 & IT259447 & Japan & $\mathrm{R}$ & $\mathrm{R}$ & $S$ & $\mathrm{~S}$ & 64 & IT101958 & Philippines & $\mathrm{R}$ & $\mathrm{R}$ & $\mathrm{R}$ & $\mathrm{R}$ & 96 & IT219266 & Unknown & $\mathrm{R}$ & $\mathrm{R}$ & $\mathrm{R}$ & $\mathrm{S}$ \\
\hline
\end{tabular}


Table 2. Annotation of candidate genes anchored by the SNPs associated with bacterial leaf blight in rice.

\begin{tabular}{|c|c|c|c|c|c|c|c|c|c|}
\hline $\begin{array}{l}\text { Races of } \\
\text { Xoo }\end{array}$ & Chr. & Position & MAF & PIC & $\begin{array}{l}\text {-Log10 } \\
(P \text {-value })\end{array}$ & $P$-value & SNP location \& type & Rice gene loci & Annotated gene function \\
\hline \multirow[t]{8}{*}{ K1 } & 9 & $10545674 *$ & 0.42 & 0.37 & 7.45 & 0.00000003 & intergenic & & unknown \\
\hline & 9 & 10742302 & 0.43 & 0.37 & 5.71 & 0.0000019 & intergenic & & unknown \\
\hline & 9 & 10899580 & 0.49 & 0.37 & 5.61 & 0.0000024 & CDS & LOC_Os09g 17810 & Leucine zipper protein-like, putative, expressed \\
\hline & 9 & 10901181 & 0.46 & 0.37 & 5.52 & 0.0000030 & intron & LOC_Os09g 17820 & Hypothetical protein \\
\hline & 11 & 1539080 & 0.08 & 0.13 & 9.56 & 0.00000278 & CDS & LOC_Os1 1 1 03860 & Ser/Thr protein kinase putative, expressed \\
\hline & 11 & 17984116 & 0.06 & 0.11 & 6.06 & 0.0000009 & Intergenic & & unknown \\
\hline & 11 & $22801669^{*}$ & 0.3 & 0.33 & 6.79 & 0.0000002 & UTR & LOC_Os11g38480 & NBS-LRR type disease resistance protein, purtative \\
\hline & 11 & 22908256 & 0.07 & 0.11 & 5.11 & 0.0000079 & CDS & LOC_Os11g38630 & Expressed protein \\
\hline \multirow[t]{17}{*}{$\mathrm{K} 2$} & 2 & 35216974 & 0.49 & 0.37 & 5.53 & 0.0000029 & CDS & LOC_Os02g57460 & RING-H2 finger protein ATL5G, putative, expressed \\
\hline & 2 & 35217262 & 0.32 & 0.34 & 5.79 & 0.0000016 & CDS & LOC_Os02g57460 & RING-H2 finger protein ATL5G, putative, expressed \\
\hline & 2 & $35236528^{*}$ & 0.38 & 0.36 & 6.5 & 0.00000026 & CDS & LOC_Os02g57510 & SNARE domain containing protein, putative, expressed \\
\hline & 2 & 35236595 & 0.45 & 0.36 & 5.57 & 0.0000099 & UTR & LOC_Os02g57520 & DNA binding protein, putative, expressed \\
\hline & 2 & 35242241 & 0.4 & 0.37 & 6.20 & 0.0000027 & Intron & LOC_Os02g57520 & DNA binding protein, putative, expressed \\
\hline & 2 & 35242550 & 0.4 & 0.36 & 6.20 & 0.0000006 & Intergenic & & unknown \\
\hline & 2 & 35251345 & 0.39 & 0.36 & 6.54 & 0.0000006 & CDS & LOC_Os02g57560 & Tyrosine protein kinase domain containing protein, putative \\
\hline & 2 & 35264051 & 0.39 & 0.36 & 5.84 & 0.0000003 & CDS & LOC_Os02g57590 & rRNA 2-o-methyletransferase fibrillarin 2, putative, ex[ressed \\
\hline & 2 & 35272592 & 0.39 & 0.36 & 6.15 & 0.0000015 & intergenic & & unknown \\
\hline & 11 & 2763639 & 0.3 & 0.33 & 5.29 & 0.0000052 & Intron & LOC_Os11g05880 & Exo 70 exocyst complex subunit, putative, expressed \\
\hline & 11 & 27090877 & 0.19 & 0.26 & 5.25 & 0.0000056 & Intergenic & & unknown \\
\hline & 11 & 28007508 & 0.16 & 0.23 & 5.71 & 0.0000019 & CDS & LOC_Os11g46250 & Expressed protein \\
\hline & 11 & 28007543 & 0.16 & 0.23 & 5.71 & 0.0000019 & CDS & LOC_Os11 1 g 46250 & Expressed protein \\
\hline & 11 & 28007573 & 0.16 & 0.23 & 5.71 & 0.0000019 & CDS & LOC_Os11g46250 & Expressed protein \\
\hline & 11 & $28753417 *$ & 0.24 & 0.30 & 7.46 & 0.000000035 & intergenic & & unknown \\
\hline & 11 & 28791082 & 0.16 & 0.23 & 7.07 & 0.0000001 & Intergenic & & unknown \\
\hline & 11 & 28791142 & 0.16 & 0.23 & 7.07 & 0.0000001 & intergenic & & unknown \\
\hline \multirow[t]{4}{*}{$\mathrm{K} 3$} & 2 & 11742061 & 0.19 & 0.26 & 4.45 & 0.000035 & Intergenic & & unknown \\
\hline & 5 & 18012531 & 0.44 & 0.37 & 4.24 & 0.0000472 & intron & LOC_Os05g31000 & $\begin{array}{l}\text { Nascent polypeptide-associated complex subunit alpha, } \\
\text { putative, expressed }\end{array}$ \\
\hline & 6 & $22800070^{*}$ & 0.12 & 0.19 & 5.19 & 0.0000064 & CDS & LOC_Os06g38470 & Histone deacetylase 19 , putative, expressed \\
\hline & 11 & $27582349^{*}$ & 0.18 & 0.25 & 4.00 & 0.00010 & CDS & LOC_Os 1 lg45570 & Expressed protein \\
\hline \multirow[t]{10}{*}{ K3a } & 4 & 31082707 & 0.48 & 0.37 & 4.18 & 0.0000654 & Intergenic & & unknown \\
\hline & 4 & $31096106^{*}$ & 0.45 & 0.37 & 4.52 & 0.000030 & $\mathrm{CDS}$ & LOC_Os04g52330 & expressed protein \\
\hline & 4 & 31096212 & 0.46 & 0.37 & 4.45 & 0.0000300 & CDS & LOC_Os04g52330 & Expressed protein \\
\hline & 4 & 31450434 & 0.39 & 0.36 & 4.08 & 0.0000824 & intron & LOC_Os04g52820 & Expressed protein \\
\hline & 11 & 8200909 & 0.11 & 0.18 & 4.03 & 0.0000942 & CDS & LOC_Os $1 \lg 14570$ & Expressed protein \\
\hline & 11 & 25395529 & 0.09 & 0.16 & 4.05 & 0.0000897 & Intron & LOC_Os1 1g42160 & F-box/LRR-repeat protein 3, putative, expressed \\
\hline & 11 & $26053709^{*}$ & 0.11 & 0.17 & 4.43 & 0.000037 & intergenic & & unknown \\
\hline & 12 & 6748428 & 0.14 & 0.21 & 4.94 & 0.0000114 & Intergenic & & unknown \\
\hline & 12 & $6895958^{*}$ & 0.18 & 0.25 & 5.25 & 0.0000056 & intron & LOC_Os12g12514 & NADP-dependent oxidoreductase, putative, expressed \\
\hline & 12 & 7130001 & 0.12 & 0.18 & 4.04 & 0.0000902 & intergenic & & unknown \\
\hline
\end{tabular}




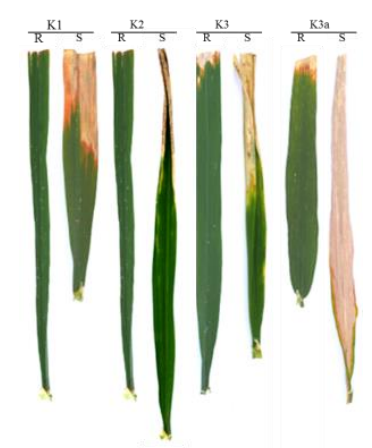

Fig 1. Bacterial leaf blight reactions to Korean isolates of Xoo, K1, K2, K3, and K3a in different accessions of rice (Oryza sativa L.). $\mathrm{R}$ and $\mathrm{S}$ stands for resistant (lesion length less than $1 \mathrm{~cm}$ ) and susceptible (lesion length more than $9 \mathrm{~cm}$ ), respectively.

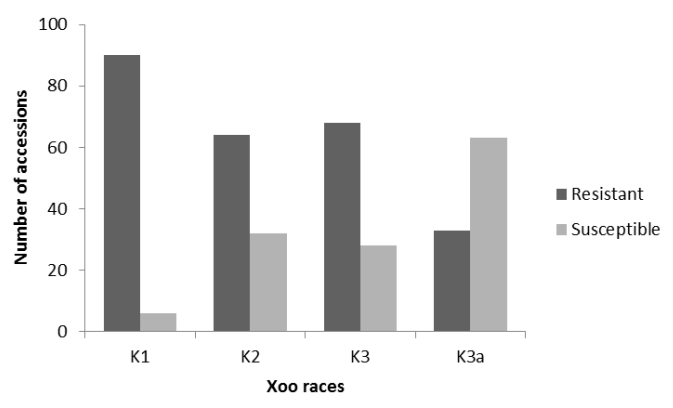

Fig 2. Distribution of rice accessions for resistance to different races of Xoo.
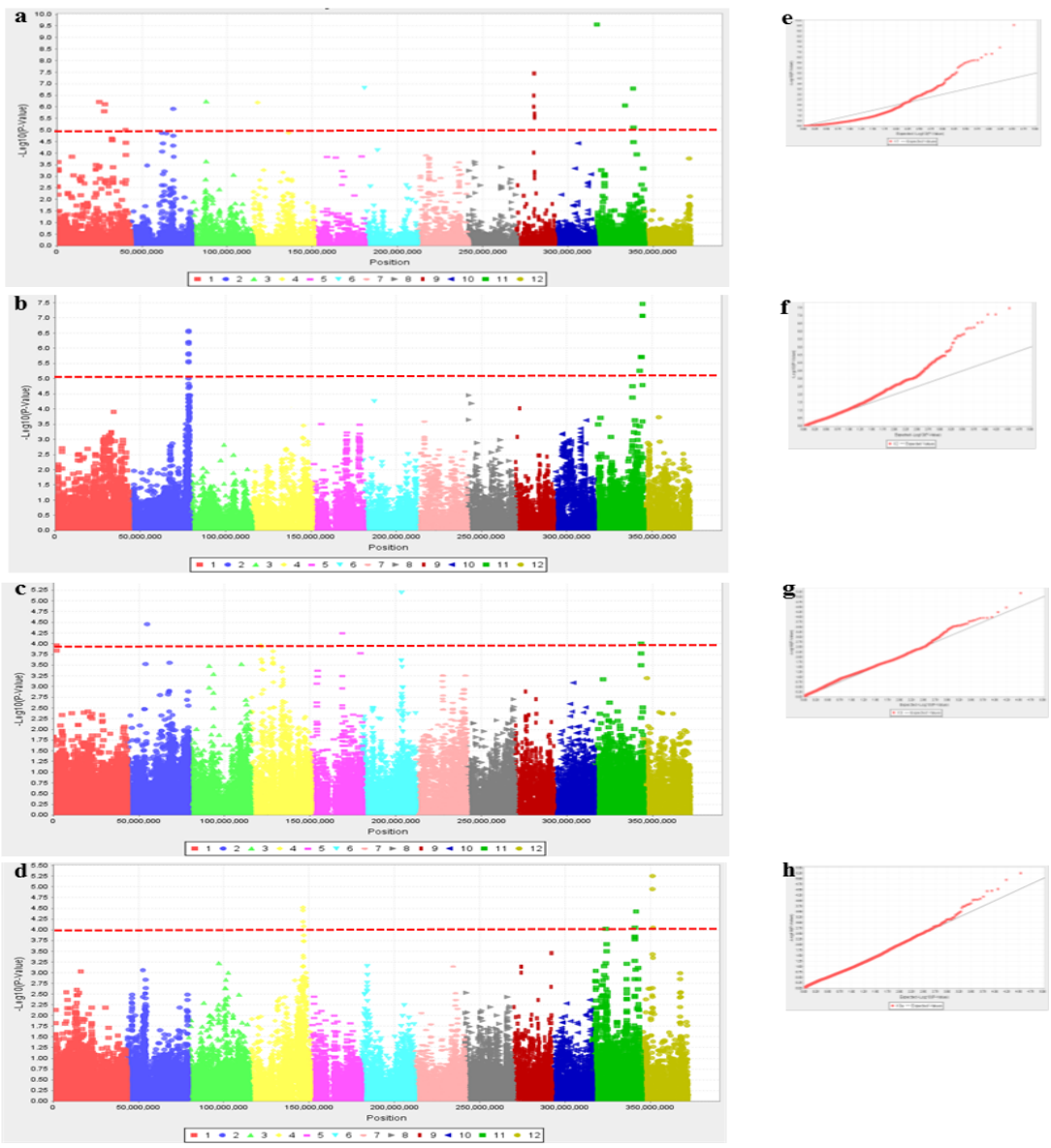

Fig 3. Bacterial leaf blight - Manhattan plots (MLM) and Quantile-Quantile plots (Q-Q) showing GWA to Xoo strains (a, e) K1, (b, f) $\mathrm{K} 2,(\mathrm{c}, \mathrm{g}) \mathrm{K} 3$, and (d, h) K3a. A high level of association of SNPs was detected on chromosome 2, 9 and 11 (a, b) and chromosome 4, 6,11 and 12 (c, d); x-axis - position on chromosomes 1 to 12; y-axis - $\log 10$ ( $P$-value) of markers; Dashed line shows significance threshold. 


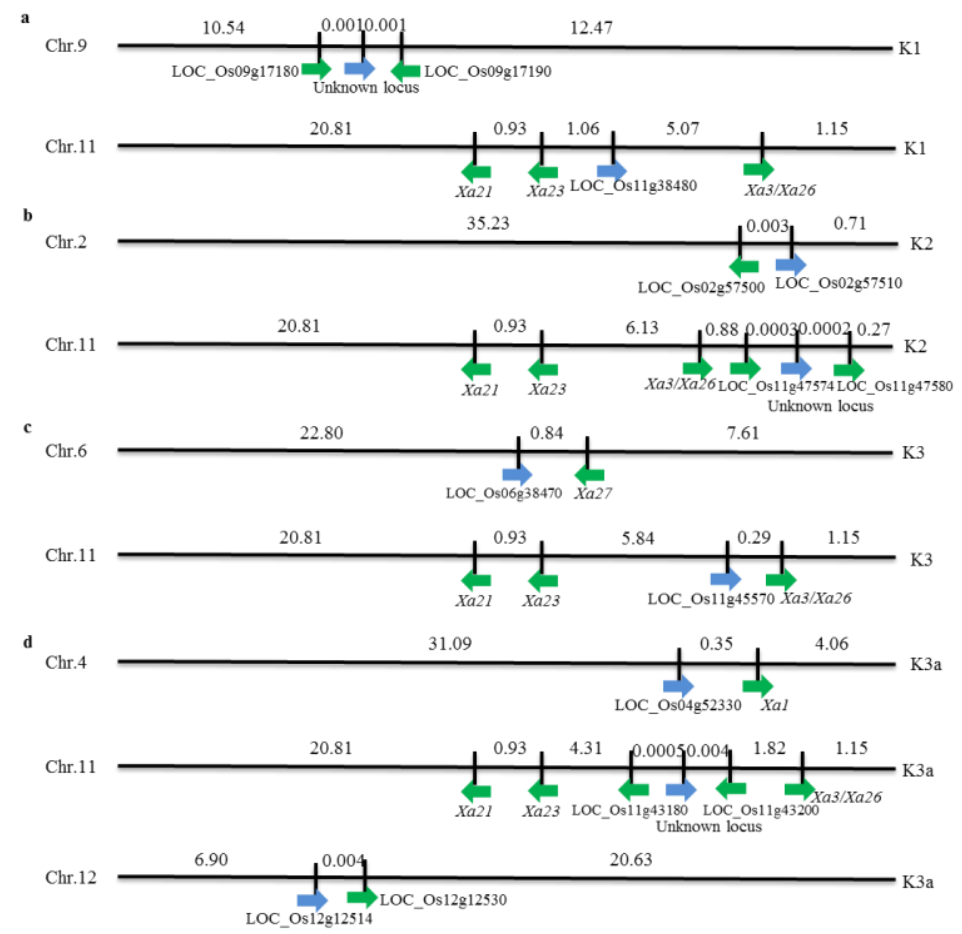

Fig 4. Diagramatic view of BLB associated (putative) loci. Location of loci on chromosome 9 and 11 against race K1 (a), chromosome 2 and 11 against race K2 (b), chromosome 6 and 11 against race K3 (c), chromosome 4, 11 and 12 against race K3a (d). The black bar indicates the chromosome band and numbers above this bar shows the approximate distance in Mb. The green arrows show the direction and position of already reported genes and blue arrows represent the new identified loci.

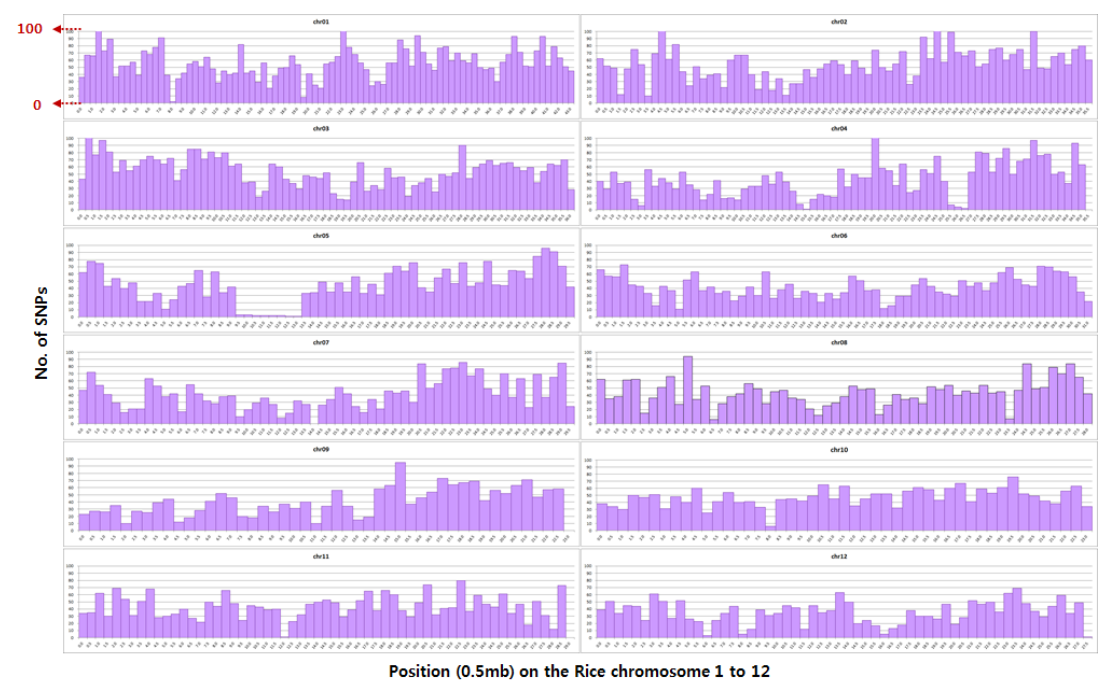

Fig 5. Distribution of SNPs in the 12 rice chromosomes. The $x$-axis represents the physical distance along each chromosome and yaxis indicates the number of SNPs.

RiceGe database (www.signal.salk.edu/cgi-bin/RiceGE) to find their positions on the chromosomes. The locus LOC_Os11g38480 (NBS-LRR type disease resistance protein, putative), which showed resistance to $\mathrm{K} 1$ race was located at a distance of $\sim 1.06 \mathrm{Mb}$ from Xa23 gene and $\sim 5.07 \mathrm{Mb}$ from Xa3/Xa26 genes on chromosome 11 at a position of $\sim 22.80 \mathrm{Mb}$. An unknown locus detected for resistance to $\mathrm{K} 1$ was located on chromosome 9 between LOC_Os09g17180 and LOC_Os09g17190 at a distance of $\sim 0.001 \mathrm{Mb}$ from each of the genes (Fig. 4a, Table 2). The resistance against K2 was indicated by a putatively BLB associated locus
LOC_Os02g57510 (SNARE domain containing protein, putative, expressed) on chromosome 2 at a distance of $\sim 0.003$ $\mathrm{Mb}$ from the LOC_Os02g57500. Similarly, a locus which showed resistance against $\mathrm{K} 2$ race was located on chromosome 11 between LOC_Os11g47580 (Glycosyl hydrolase, putative) and LOC_Os11g47574 (Expressed protein). The Xa3/Xa26 and an unknown locus were located $\sim 0.88 \mathrm{Mb}$ apart on the same chromosome (Fig. 4b). Another putative locus LOC_Os11g45570 (expressed protein), which showed resistance to $\mathrm{K} 3$ race, was found between $\mathrm{Xa23}$ and $\mathrm{Xa3} / \mathrm{Xa26}$ genes at a distance of $\sim 5.84 \mathrm{Mb}$ and $\sim 0.29 \mathrm{Mb}$, respectively. 
However, the direction of $X a 23$ was opposite to LOC_Os11g45570 and Xa3/Xa26 genes (Fig. 4c). The Xa3 and $X a 26$ genes were shown together because of the presence of genetically tight linkage between $\mathrm{Xa3}$ gene and a leucine-rich repeat (LRR) gene Xa26 (Xiang et al., 2006). Another locus LOC_Os04g52330 (expressed protein), which indicated resistance to $\mathrm{K} 3 \mathrm{a}$ race, was located at a distance of $\sim 0.35 \mathrm{Mb}$ from $\mathrm{Xal}$ gene on chromosome 4. One more unknown locus putatively associated with BLB resistance was located between LOC_Os11g43180 (Expressed protein) and LOC_Os11g43200 (Tropinone reductase 2). Resistance to $\mathrm{K} 3 \mathrm{a}$ race was also indicated by LOC_Os12g12514 on chromosome 12 at the position of $\sim 6.90 \mathrm{Mb}$ and at a distance of $\sim 0.004 \mathrm{Mb}$ from LOC_Os12g12530 (Retrotransposon protein, putative) (Fig. 4d, Table 2).

\section{Discussion}

In the past, numerous studies have been conducted on BLB disease related diagnosis, management, and control, but no effective and economical treatment has been established. Nevertheless, improving genetic resistance is a competent way of combating disease. This can be achieved by screening genotypes conferring resistance against pathogens. Previously, Ali et al. (2009) determined five out of 15 genotypes against $X$. oryzae. In another study, three basmati rice varieties were screened against eight different isolates of Xoo to test the resistance level of commercially grown cultivars in Pakistan. However, these varieties proved to be susceptible to pathogens (Noor et al., 2006). Similarly, 53 medium-grain and 49 fine grain rice cultivars were employed for screening against BLB races, of which 13 for medium-grain and one for fine grain were selected as resistant cultivars (Yasin et al., 2007). It is vital to have more knowledge of varietal resistance for selecting cultivars with durable resistance (Banito et al., 2010). In the present study, 96 accessions were screened against four different Korean races of Xoo (K1, K2, K3, and K3a) and found that all the accessions were resistant to at least one race (Table 1). Previously, Fred et al. (2016) identified five resistant cultivars out of 32 cultivars against $\mathrm{K} 1$ race and reported the severity of pathogen in the field conditions. However, germplasm accessions used in our study proved to be strongly resistant $(93.75 \%)$ to K1 race. Furthermore, $22.92 \%$ accessions showed resistance to four races of Xoo. Among the four races, high rate of susceptibility (65.62\%) in germplasm was observed for K3a. Though $\mathrm{K} 3 \mathrm{a}$ is prevailing as a threat to rice yield in Korea (Noh et al., 2003), three accessions from Korean germplasm (IT260672, IT219216, and IT219282) showed resistance against all tested races including K3a. These findings suggest that the resistant germplasm may contain multiple resistance genes or QTLs, or a single locus may confer resistance to multiple races.

During an attack from a pathogen, plants are protected by defensive signaling pathways (Koornneef and Pieterse, 2008) and many genes that are essential during infection start expressing. Durable resistance to BLB is a complex trait and involves both dominant and recessive genes. One strategy to achieve long term resistance to BLB is to accumulate QTLs that confer broad spectrum resistance. In this study, we inoculated 96 germplasm accessions with four isolates from Korea and phenotypic results were used in GWAS by using 34,724 SNPs. With the help of Manhattan plots at a threshold level of $[-\log 10(P$-value $)]$ more than 5 (Fig. 3a, 3b) and more than 4 (Fig 3c, 3d), nine loci putatively linked to BLB were identified. The effects of these loci on resistance to BLB might be as strong as those of other resistance genes. Although the functions of the candidate QTL associated with BLB require confirmation, detection of loci associated with resistance against Korean populations of $X$. oryzae provides basis for detailed molecular analysis. Furthermore, some of the SNPs in these loci with high linear $-\log 10(P$-value $)$ could be used as a marker in the marker assisted selection of BLB resistant cultivars in Korea.

Among the identified loci in the present study, LOC_Os11g38480, LOC_Os02g57510, LOC_Os06g38470, LOC_Os04g52330, and LOC_Os12g12514 including an unknown locus on chromosome 9 showed strong associations with BLB resistance conferred for $\mathrm{K} 1, \mathrm{~K} 2, \mathrm{~K} 3$, and $\mathrm{K} 3 \mathrm{a}$, respectively. Our search of the reference 'Oryza sativa japonica' genomic sequence revealed that an unknown locus, which showed resistance against $\mathrm{K} 1$ race was positioned between a hypothetical protein (LOC_Os09g17180) and OsFBX320-Fbox domain containing protein (LOC_Os09g17190). F-box domain plays its role in the degradation of cellular proteins. Though there is no evidence linked to BLB resistance, FBX proteins have been reported in almost all terrestrial species which perform different functions essential to life on land including defense against pathogens (Hua et al., 2011). Similarly, a gene (LOC_Os11g45570) resistant to K3 race and an unknown locus resistant to K2 race were localized near $X a 3 / X a 26$ genes on chromosome 11. The unknown locus with resistance against $\mathrm{K} 2$ race was positioned between an expressed protein and glycosyl hydrolase, putative gene on chromosome 11. The glycosyl hydrolase family has been reported for important physiological processes, including response to biotic and abiotic stresses in plants (Opassiri et al., 2006) (Fig. 3b). Among other nearest genes, Xa3/Xa26 have been reported for resistance against three races, including $\mathrm{K} 1$, $\mathrm{K} 2$, and $\mathrm{K} 3$ but susceptible to $\mathrm{K} 3 \mathrm{a}$ (Kim et al., 2015). Moreover, Suh et al. (2009) reported that plants having Xa4 and $\mathrm{Xa} 21$ genes conferred strong resistance to $\mathrm{K} 3 \mathrm{a}$ race. Another unknown locus on chromosome 11 for K3a race was located between an expressed protein and tropinone reductase 2 gene, which helps in oxidoreductase activity in the cells. Additionally, the positions of Xa21,Xa23 and Xa3/Xa26 genes near to an unknown locus, identified in this study on chromosome 11 , indicated an association for resistance to $\mathrm{K} 3 \mathrm{a}$ race (Fig. 3d). It suggests that expressed protein (LOC_Os11g45570) and unknown regions could be exploited as candidate resistance loci for K2, K3 and K3a races in Korea. Similarly, another expressed protein (LOC_04g52330) on chromosome 4 was localized close to $\mathrm{Xal}$ gene, which has resistance against $\mathrm{K} 1$, $\mathrm{K} 2$, and $\mathrm{K} 3$ races (Kim et al., 2015). Interestingly the locus identified in this study on chromosome 4 showed resistance against $\mathrm{K} 3 \mathrm{a}$ race, which might be useful to harness it as a new resistance locus for K3a race. Most of the resistance responsive genes are anchored on chromosome 11 and 12, as depicted in the present and a previous study (Rice and Sequencing, 2005). Therefore, these chromosomes specifically yield a desirable target for breeding durable disease resistance in rice.

\section{Materials and Methods}

\section{Plant materials}

A total of 96 accessions of rice germplasm were selected on the basis of the bioassay using four isolated races of Xoo such as $\mathrm{K} 1, \mathrm{~K} 2, \mathrm{~K} 3$ and $\mathrm{K} 3 \mathrm{a}$. All genotypes of rice were acquired from National Agrobiodiversity Center (NAS, RDA, Republic of Korea). The detailed information about the plant material used in this study is given in Table 1. The seeds were sown in green house and seedlings were transplanted in the field (21 days after sowing) with a planting density of $30 \times 15 \mathrm{~cm}$. The experiment was performed with three replications. Separate 
plots were used for different isolates and management practices in the field were as usual.

\section{Bioassay for bacterial leaf blight strains}

Korean BLB isolates have been classified into five races (K1 to K5) on the basis of five rice cultivars including Milyang 42, Hangangchalbyeo, Pungsanbyeo, Cheongcheongbyeo, and Milyang 23 as the Xoo differential system (Yun et al., 1985). In 2003, a new race K3a emerged in Korea which proved to be an epidemic for rice crop (Noh et al., 2003). Among these BLB isolates, four races K1 (HB01013), K2 (HB01014), K3 (HB01015), and K3a (HB01009) (Song et al., 2014) that were maintained at $-80^{\circ} \mathrm{C}$ were revived on Peptone sucrose agar (PSA) plates at $28^{\circ} \mathrm{C}$ for $48 \mathrm{~h}$. Each bacterial colony was suspended with sterilized distilled water and adjusted to concentrations of approximately $10^{9} \mathrm{cfu} / \mathrm{ml}$ (Fang et al., 1981). At the booting stage (approximately 40 days after transplanting), the uppermost fully expanded leaves of each plant were inoculated by clipping the scissors in bacterial suspension and by clipping off the leaves $2-3 \mathrm{~cm}$ from leaf tip (Kauffman et al., 1973). The inoculations were performed in the morning in order to reduce the possible effects of high temperature.

\section{Disease reaction}

For disease scoring, the lesion length was measured 28 days after inoculation (DAI) from the leaf tips. The plant leaves with lesion length less than $1 \mathrm{~cm}$ were selected as resistant and those with more than $9 \mathrm{~cm}$ were selected as susceptible.

\section{Genotyping}

Young leaves from two week old plantlets were used for DNA extraction. Genomic DNA was extracted according to the Qiagen DNeasy Plant Mini Kit protocol (QIAGEN, Germany). The concentrations of DNA were estimated using Take $3^{\mathrm{TM}}$ Micro-Volume Plate (BioTek Instruments, Inc., USA) and final adjustment was made at $100 \mathrm{ng} / \mu \mathrm{l}$. The protocol of Elshire et al. (2011) was used to prepare 384-plex genotyping by sequencing (GBS) libraries and Illumina HiSeq 2000 paired-end read was used for GBS sequencing. The discovered SNPs were called from the 384-plex GBS data using TASSEL 5.0 GBS pipeline (Bradbury et al., 2007) with physical alignment to the reference genome, Oryza sativa L. ssp. Japonica (https://phytozome.jgi.doe.gov/) using Bowtie2 (Spindel et al., 2013). Imputation of missing data was performed in TASSEL 5.0 using FastImputation-BitFixedWindow plugin with default settings (Romay et al., 2013). The algorithm divides the whole SNP set into small SNP windows and identifies the most similar accession within each window to fill the missing data. The data point is left missing if the window from nearest neighbor has difference of $>5 \%$ from the accession being imputed (Romay et al., 2013). The average imputation error rate across the 12 chromosomes was estimated to be less than $1 \%$ by comparing the imputed and actual calls. Total SNP matrix was obtained for a total of 293,379 SNPs. All those SNPs were removed from the dataset which had $10 \%$ or more missing data after imputation. Finally, a dataset of 34,724 SNPs was obtained, which expanded from chromosome one to twelve in the rice genome (Fig. 5).

\section{Association mapping}

GWAS was conducted using TASSEL 5.0. The TASSEL program uses a mixed linear model (MLM), which includes a kinship matrix in addition to any covariates to determine association between traits and phenotype. In our case, we described disease resistance as a simple binary trait where 1 and 0 represented susceptible and resistant trait, respectively. The final analysis used six principal components as covariates along with the kinship matrix in the MLM. Statistically significant loci were identified by applying a BY-FDR (false discovery rate) correction for multiple tests (Benjamini and Yekutieli, 2001). Allele effects were calculated as the difference between the average trait value for all accessions that were homozygous for the major allele (AA) and the average trait value for all accessions that were homozygous for the minor allele (BB) for a given SNP. The percent variance explained by each individual significant SNP was calculated as the squared correlation between the phenotype and genotype of the SNP (Faraway, 2002). Q-Q (Quantile-Quantile) plots and Manhattan plots were generated using TASSEL 5.0

\section{Conclusion}

The present investigation revealed the existence of high resistance in 22 accessions against four tested Korean isolates of $X$. oryzae. Among these accessions, Japanese germplasm was more susceptible, whereas Philippines germplasm was more resistant. Furthermore, three Korean accessions showed strong resistance to the four races of Xoo. These accessions may contain multiple resistance genes or QTL that are effective against Korean races. Crossing these resistant germplasm accessions with susceptible cultivars in Korea will facilitate the resistance breeding against BLB. The SNPs reported here can be used to develop functional markers for marker-assisted selection of resistant genotypes. Moreover, functional characterization of the putative loci could be performed for elucidating their biological role in BLB resistance.

\section{Acknowledgement}

This study was supported by a grant (Code no. PJ010106) from the National Institute of Agricultural Sciences, RDA, Republic of Korea.

\section{References}

Adhikari TB, Vera Cruz CM, Zhang Q, Nelson RJ, Mew TW, Leach JE (1995) Genetic diversity of Xanthomonas oryzae pv. oryzae in Asia. Appl Environ Microbiol. 61:966-971.

Ali A, Khan MH, Bano R, Rashid H, Raja NI, Chaudhry Z (2009) Screening of Pakistani rice (Oryzae sativa) cultivars against Xanthomonas oryza pv. oryzae. Pak J Bot. 41:25952604.

Banito A, Kpemoua KE, Wydra K (2010) Screening of rice genotypes for resistance to bacterial blight using strain $\mathrm{x}$ genotype interactions. J Plant Pathol. 92:181-186.

Benjamini Y, Yekutieli D (2001) The control of the false discovery rate in multiple testing under dependency. Ann Stat. 29:1165-1168.

Bradbury PJ, Zhang Z, Kroon DE, Casstevens TM, Ramdoss Y, Buckler ES (2007) TASSEL: software for association mapping of comples traits in diverse samples. Bioinformatics. 23:2633-2635.

Buckler ES, Holland JB, Bradbury PJ, Acharya CB, Brown PJ, Browne C, Ersoz E, Flint-Garcia S, Garcia A, Glaubitz JC, Goodman MM, Harjes C, Guill K, Karoon DE, Larsson S, Lepak NK, Li H, Mitchell SE, Pressoir G, Peiffer JA, Rosas MO, Rocheford TR, Romay MC, Romero S, Salvo S, Villeda HS, da Silva HS, Sun Q, Tian F, Upadyayula N, Ware D, Yates H, Yu J, Zhang Z, Kresovich S, McMullen MD (2009) 
The genetic architecture of maize flowering time. Science. 325:714-718.

Chen H, Wang S, Zhang Q (2002) A new gene for bacterial blight resistance in rice located on chromosome 12 identified from Minghui 63 and elite restorer line. Phytopathology. 92:750-754

Chen S, Liu X, Zeng L, Ouyang D, Yang J, Zhu X (2011) Genetic analysis and molecular mapping of a novel recessive gene $x a 34(t)$ for resistance against Xanthomonas oryzae pv. oryzae. Theor Appl Genet. 122:1331-1338.

Das B, Sengupta S, Prasad M, Ghose TK (2014) Genetic diversity of the conserved motifs of six bacterial leaf blight resistance genes in a set of rice landraces. BMC Genet. 15:82.

Elshire RJ, Glaubitz JC, Sun Q, Poland JA, Kawamoto K, Buckler ES, Mitchell SE (2011) A robust, simple genotypingby-sequencing (GBS) approach for high diversity species. PLoS One. 6:e19379.

Fang ZD, Xu ZG, Guo CJ, Chen YL, Deng XR, Li HS, Zhu LM (1981) Variability of pathogenicity of rice bacterial leaf blight organism. J Nanjing Agr Technol Coll. 1:1-11.

Faraway JJ (2002) Practical regression and anova using R. http://www.stat.lsa.umich.edu/ faraway/book/pra.pdf

Fred AK, Kiswara G, Yi G, Kim KM (2016) Screening rice cultivars for resistance to bacterial leaf blight. J Microbiol Biotechnol. 26(5):938-945.

Huang X, Wei X, Sang T, Zhao Q, Feng Q, Zhao Y, Li C, Zhu C, Lu T, Zhang Z, Li M, Fan D, Guo Y, Wang A, Wang L, Deng L, Li W, Lu Y, Weng Q, Liu K, Huang T, Zhou T, Jing Y, Li W, Lin Z, Buckler ES, Qian Q, Zhang QF, Li J, Han B (2010) Genome-wide association studies of 14 agronomic traits in rice land races. Nat Genet. 42:961-967.

Hua Z, Zou C, Shiu SH, Vierstra RD (2011) Phylogenetic comparison of $F-B o x(F B X)$ gene superfamily within the plant kingdom reveals divergent evolutionary histories indicative of genomic drift. PLoS One. 6(1):e16219.

Jeung JU, Heu SG, Shin MS, Vera Cruz CM, Jena KK (2006) Dynamics of Xanthomonas oryzaepv. oryzae populations in Korea and their relationship to known bacterial blight resistance genes. Phytopathology. 96:867-875.

Jia G, Huang X, Zhi H, Zhao Y, Zhao Q, Li W, Chai Y, Yang L, Liu K, Lu H, Zhu C, Lu Y, Zhou C, Fan D, Weng Q, Guo Y, Huang T, Zhang L, Lu T, Feng Q, Hao H, Liu H, Lu P, Zhang N, Li Y, Guo E, Wang S, Wang S, Liu J, Zhang W, Chen G, Zhang B, Li W, Wang Y, Li H, Zhao B, Li J, Diao X, Han B (2013) A haplotype map of genomic variations and genome wide association studies of agronomic traits in foxtail millet (Setaria italica). Nat Genet. 45:957-961.

Kauffman HE, Reddy APK, Hsieh SPY, Merca SD (1973) An improved technique for evaluating resistance of rice varieties to Xanthomonas oryzae. Plant Dis Rep. 57:537-541.

Khan MA, Naeem M, Iqbal M (2014) Breeding approaches for bacterial leaf blight resistance in rice (Oryza sativa L.), current status and future directions. Eur $\mathrm{J}$ Plant Pathol. 139:27-37.

Khush GS, Bacalangco E, Ogawa T (1990) A new gene for resistance to bacterial blight from $O$. longistaminata. Rice Genet Newsl. 7:121-122.

Kim SM, Suh JP, Qin Y, Noh TH, Reinke RF (2015) Identification and fine-mapping of a new resistance gene, $X a 40$, conferring resistance to bacterial blight races in rice (Oryza sativa L.). Theor Appl Genet. 128:1933-1943.

Kim SI, Song JT, Jeong JY, Seo HS (2016) Niclosamide inhibits leaf blight caused by Xanthomonas oryzae in rice. Sci Rep. 6:21209. doi: 10.1038/srep21209

Kump KL, Bradbury PJ, Wisser RJ, Buckler ES, Belcher AR, Oropeza-Rosas MA, Zwonitzer JC, Kresovich S, McMullen MD, Ware D, Balint-Kurti PJ, Holland JB
(2011) Genome wide association study of quantitative resistance to southern leaf blight in the maize nested association mapping population. Nat Genet. 43:163-168.

Koornneef A, Pieterse CM (2008) Cross talk in defense signaling. Plant Physiol. 146:839-844.

Lee KS, Rasabandith S, Angeles ER, Khush GS (2003) Inheritance of resistance to bacterial blight in 21 cultivars of rice. Phytopathology. 93:147-152.

Mew TW (1987) Current status of future prospects of research on bacterial blight of rice. Annu Rev Phytopathol. 25:359382.

Nelson RJ, Baraoidan MR, Vera Cruz CM, Yap IV, Leach JE, Mew TW, Leung H (1994) Relationship between phylogeny and pathotype for the bacterial blight pathogen of rice. Appl Environ Microbiol. 60:3275-3283.

Noh TH, Lee DK, Kang MH, Shin MS, Na SY (2003) Identification of new race of Xanthomonas oryzae pv. oryzae (Xoo) in Korea. (Abstr.) Phytopathology. 93:S66.

Noh TH, Lee DK, Park JC, Shim HK, Choi MY, Kang MH, Kim JD (2007) Effects of bacterial blight occurrence on rice yield and grain quality in different rice growth stage. Res Plant Dis. 13:20-23.

Noor A, Chaudhry Z, Rashid H, Mirza B (2006) Evaluation of resistance of rice varieties against bacterial blight caused by Xanthomonas oryzae pv. oryzae. Pak J Bot. 38:193-203.

Nordborg M, Weigel D (2008) Next-generation genetics in plants. Nature. 456:720-723.

Ogawa T (1996) Monitoring race distribution and identification of genes for resistance to bacterial leaf blight. In rice genetics III, proceeding of the $3^{\text {rd }}$ international rice genetics symposium. edited by Khush GS. International Rice Research Institute, Manila, Philippines. pp.456-459.

Opassiri R, Pomthong B, Onkoksoong T, Akiyama T, Esen A, Ketudat-Cairns JR (2006) Analysis of rice glycosyl hydrolase family 1 and expression of Os4bglu $12 \beta$-glucosidase. BMC Plant Biol. 6:33.

Poland JA, Bradbury PJ, Buckler ES, Nelson RJ (2011) Genome-wide nested association mapping of quantitative resistance to northern leaf blight in maize. Proc Natl Acad Sci USA. 108:6893-6898.

Rice C, Sequencing C (2005) The sequence of rice chromosomes 11 and 12, rich in disease resistance genes and recent gene duplications. BMC Biol. 3:20. doi: 10.1186/1741-7007-3-20

Romay MC, Millard MJ, Glaubitz JC, Peiffer JA, Swarts KL, Casstevens TM, Elshire RJ, Acharya CB, Mitchell SE, FlintGarcia SA, McMullen MD, Holland JB, Buckler ES, Gardner CA (2013) Comprehensive genotyping of the USA national maize inbred seed bank. Genome Biol. 14:R55. doi: 10.1186/gb-2013-14-6-r55

Sakaguchi S (1967) Linkage studies on the resistance to bacterial leaf blight, Xanthomonas oryzae (Uyeda et Ishiyama) dowson, in rice. Bull Natl Inst Agr Sci Ser. D16:1-18. (in Japanese with English summary)

Song ES, Kim SY, Noh TH, Cho H, Chae SC, Lee BM (2014) PCR based assay for rapid and specific detection of the new Xanthomonas oryzae pv. oryzae $\mathrm{K} 3 \mathrm{a}$ race using an AFLPderived marker. J Microbiol Biotechn. 24(6):732-739.

Suh JP, Noh TH, Kim KY, Kim JJ, Kim YG, Jena KK (2009) Expression levels of three bacterial blight resistance genes against $\mathrm{K} 3 \mathrm{a}$ race of Korea by molecular and phenotype analysis in japonica rice (O. sativa $\mathrm{L}$.). J Crop Sci Biotech. 12(3):103-108.

Spindel J, Wright M, Chen C, Cobb J, Gage J, Harrington S, Lorieux M, Ahmadi N, McCouch S (2013) Bridging the genotyping gap: using genotyping by sequencing (GBS) to 
add high-density SNP markers and new value to traditional bi-parental mapping and breeding populations. Theor Appl Genet. 126(11):2699-2716.

Taura S, Ogawa T, Yoshimura A, Ikeda R, Iwata N (1992) Identification of a recessive resistance gene to rice bacterial blight of mutant line XM6, Oryzae sativa L. Jpn J Breed. 42:7-13.

Xiang Y, Cao Y, Xu C, Li X, Wang S (2006) Xa3, conferring resistance for rice bacterial and encoding a receptor kinaselike protein, is the same as Xa26. Theor Appl Genet. 113:1347-1355.

Yasin SI, Ali SS, Shah JA, Fatima K (2007) Evaluation of medium and fine grain lines/varieties against bacterial leaf blight (Xanthomonas oryzae pv. oryzae) of rice. Pak J Phytopathol. 19:206-210.

Yoshimura S, Yoshimura A, Saito A, Kishimoto N, Kawase M, Yano M, Nakagahra M, Ogawa T, Iwata N (1992) RFLP analysis of introgressed chromosomal segments in three nearisogenic lines of rice for bacterial blight resistance genes, $\mathrm{Xa}$ 1, Xa-3 and Xa-4. Jpn J Genet. 67:29-37.
Yoshimura S, Yamanouchi U, Katayose Y, Toki S, Wang ZX, Kono I, Kuruta N, Yano M, Iwata N, Sasaki T (1998) Expression of $\mathrm{Xal}$, a bacterial blight resistance gene in rice, is induced by bacterial inoculation. Proc Natl Acad Sci USA. 95:1663-1668.

Yun MS, Lee EJ, Cho YS (1985) Pathogenic specialization of the rice bacterial leaf blight pathogen, Xanthomonas campestris pv. oryzae: race classification based on reactions of Korean differential varieties. Korean J Plant Prot. 24:97101. (in Korean with English abstract)

Zhang Q, Lin SC, Zhao BY, Wang CL, Yang WC, Zhou YL, Li DY, Chen CB, Zhu LH (1998) Identifcation and tagging a new gene for resistance to bacterial blight (Xanthomonas oryzae pv. oryzae) from O. rufipogon. Rice Genet Newsl. $15: 138-142$. 